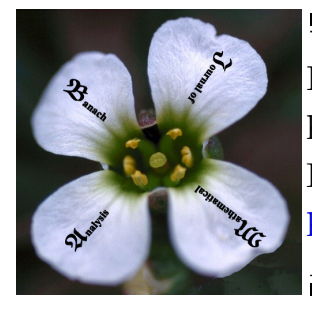

Banach J. Math. Anal. 9 (2015), no. 3, 140-152

http://doi.org/10.15352/bjma/09-3-10

ISSN: $1735-8787$ (electronic)

http://projecteuclid.org/bjma

\title{
INTERPOLATION CLASSES AND MATRIX MEANS
}

\author{
DINH TRUNG HOA ${ }^{1}$, TOAN M. HO ${ }^{2}$ AND HIROYUKI OSAKA ${ }^{3 *}$ \\ Communicated by F. Kittaneh
}

\begin{abstract}
Using a 'local' integral representation of a matrix connection of order $n$ corresponding to an interpolation function of the same order, for each integer $n$, we can describe an injective map from the class of matrix connections of order $n$ to the class of positive $n$-monotone functions on $(0, \infty)$ and the range of this corresponding covers the class of interpolation functions of order $2 n$. In particular, the space of symmetric connections is isomorphic to the space of symmetric positive $n$-monotone functions. Moreover, we show that, for each $n$, the class of $n$-connections extremely contains that of $(n+2)$-connections.
\end{abstract}

\section{INTRODUCTION}

Throughout the paper, let us denote $\mathbb{R}_{+}$the subset $(0, \infty)$ of the real line $\mathbb{R}$, $M_{n}$ the algebra of square matrices of order $n$ with coefficients in $\mathbb{C}$ and $M_{n}^{+}$the cone of positive semi-definite matrices in $M_{n}$. The order relation $A \leq B$ on the set of all self-adjoint matrices means that $B-A \geq 0$. A $n$-monotone function on $[0, \infty)$ is a function which preserves the order on the set of all $n \times n$ positive semi-definite matrices. Moreover, if $f$ is $n$-monotone for all $n \in \mathbb{N}$, then $f$ is called operator monotone.

With a view to studying electrical network connections, Anderson and Duffin [5] introduced the concept of parallel sum of two positive semi-definite matrices. Subsequently, in [6] Anderson and Trapp have extended the notions of parallel addition and shorted operation to bounded linear positive operators on a Hilbert

Date: Received: Jul. 22, 2014; Revised: Oct. 3, 2014; Accepted: Oct. 23, 2014.

* Corresponding author.

2010 Mathematics Subject Classification. Primary 46L30; Secondary 15A45.

Key words and phrases. Interpolation functions, matrix monotone functions, mean of positive matrices. 
space $H$ and showed their important applications in operator theory. In the paper [12] Kubo and Ando developed an axiomatic theory of operator means. This theory has found a number of applications in operator theory and quantum information theory. In particular, Petz [17] connected the theory of monotone metrics with the theory of operator means by Kubo and Ando. He proved that an operator monotone function $f:[0, \infty) \longrightarrow[0, \infty)$ satisfying the symmetry condition

$$
f(t)=t f\left(t^{-1}\right), \quad t \geq 0
$$

is related to a Morozova-Chentsov function which gives a monotone metric on the quantum state which consists of $n \times n$ density matrices.

Restricting the definition of operator means from [12] on the set of positive matrices of order $n$, we can consider matrix means of positive matrices of order $n$.

Definition 1.1. A binary operation $\sigma$ on $M_{n}^{+},(A, B) \mapsto A \sigma B$ is called a matrix connection of order $n$ (or $n$-connection) if it satisfies the following properties:

(I) $A \leq C$ and $B \leq D$ imply $A \sigma B \leq C \sigma D$.

(II) $C(A \sigma B) C \leq(C A C) \sigma(C B C)$.

(III) $A_{n} \downarrow A$ and $B_{n} \downarrow B$ imply $A_{n} \sigma B_{n} \downarrow A \sigma B$

where $A_{n} \downarrow A$ means that $A_{1} \geq A_{2} \geq \ldots$ and $A_{n}$ converges strongly to $A$.

A mean is a normalized connection, i.e. $1 \sigma 1=1$. An operator connection means a connection of every order. A $n$-semi-connection is a binary operation on $M_{n}^{+}$satisfying the conditions (II) and (III).

In [12], by using the representation of operator monotone functions on $[0, \infty)$, Kubo and Ando showed that there exists an affine order-isomorphism from the class of connections onto the class of positive operator monotone functions. The following natural question is one of the motivations of our study: Does there exist an injective affine order-homomorphism from the class of $n$-connections to the class of positive $n$-monotone functions on $[0, \infty)$ ? To study this question, the approach in [12] could not be used, since it is not clear if there is an integral representation of $n$-monotone functions. We need another candidates replacing $n$-monotone functions.

A function $f: \mathbb{R}_{+} \rightarrow \mathbb{R}_{+}$is called an interpolation function of order $n$ ([1]) if for any $T, A \in M_{n}$ with $A>0$ and $T^{*} T \leq 1$

$$
T^{*} A T \leq A \quad \Longrightarrow \quad T^{*} f(A) T \leq f(A) .
$$

We denote by $\mathcal{C}_{n}$ the class of all interpolation functions of order $n$ on $\mathbb{R}_{+}$.

Remark 1.2. Let $P\left(\mathbb{R}_{+}\right)$be a set of all Pick functions on $\mathbb{R}_{+}, P^{\prime}$ the set of all positive Pick functions on $\mathbb{R}_{+}$, i.e., functions of the form

$$
h(s)=\int_{[0, \infty]} \frac{(1+t) s}{1+t s} d \rho(t), \quad s>0,
$$

where $\rho$ is some positive Radon measure on $[0, \infty]$. For $n \in \mathbb{N}$ denote by $P_{n}^{\prime}$ the set of all strictly positive $n$-monotone functions. The following properties can be found in [1], [2], [3], [11], [14] or [4], : 
(i) $P^{\prime}=\cap_{n=1}^{\infty} P_{n}^{\prime}, P^{\prime}=\cap_{n=1}^{\infty} \mathcal{C}_{n}$;

(ii) $\mathcal{C}_{n+1} \subseteq \mathcal{C}_{n}$;

(iii) $P_{n+1}^{\prime} \subseteq \mathcal{C}_{2 n+1} \subseteq \mathcal{C}_{2 n} \subseteq P_{n}^{\prime}, P_{n}^{\prime} \subsetneq \mathcal{C}_{n}$

(iv) $\mathcal{C}_{2 n} \subsetneq P_{n}^{\prime}[16]$;

(v) A function $f: \mathbb{R}_{+} \rightarrow \mathbb{R}_{+}$belongs to $\mathcal{C}_{n}$ if and only if $\frac{t}{f(t)}$ belongs to $\mathcal{C}_{n}$ [4, Proposition 3.5].

The following useful characterization of a function in $\mathcal{C}_{n}$ is due to Donoghue (see [9], [8]), and to Ameur (see [1]).

Theorem 1.3. [4, Corollary 2.4] A function $f: \mathbb{R}_{+} \rightarrow \mathbb{R}_{+}$belongs to $\mathcal{C}_{n}$ if and only if for every $n$-set $\left\{\lambda_{i}\right\}_{i=1}^{n} \subset \mathbb{R}_{+}$there exists a positive Pick function $h$ on $\mathbb{R}$, such that

$$
f\left(\lambda_{i}\right)=h\left(\lambda_{i}\right) \quad \text { for } \quad i=1, \ldots, n .
$$

As a consequence, Ameur gave a 'local' integral representation of every function in $\mathcal{C}_{n}$ as follows.

Theorem 1.4. [2, Theorem 7.1] Let $A$ be a positive definite matrix in $M_{n}$ and $f \in \mathcal{C}_{n}$. Then there exists a positive Radon measure $\rho_{\sigma(A)}$ on $[0, \infty]$ such that

$$
f(A)=\int_{[0, \infty]} A(1+s)(A+s)^{-1} d \rho_{\sigma(A)}(s),
$$

where $\sigma(A)$ is the set of eigenvalues of $A$.

Applying this representation, we give a 'local' integral formula for a connection of order $n$ corresponding to a $n$-monotone function on $(0, \infty)$ (hence, an interpolation function of order $n$ ) via the formula (2.1) (Lemma 2.1). Furthermore, this 'local' formula also establishes, for each interpolation function $f$ of order $2 n$, a connection $\sigma$ of order $n$ corresponding to the given interpolation function $f$. Therefore, it shows that the map from the $n$-connections to the interpolation functions of order $n$ is injective with the range containing the interpolation functions of order $2 n$. Moreover, we also show that the class of 1-connections is isomorphic to the class of interpolation functions of order 2 and as much as properties we know in the space of $n$-connections also hold in the space $\mathcal{C}_{2 n}$ of interpolation functions of order $2 n$ (Proposition 3.1 and Proposition 2.8). This gives a hope that the class of $n$-connections is isomorphic to the class $\mathcal{C}_{2 n}$.

An interesting and well-studied class of $n$-connections is the symmetric one, since the corresponding representation functions $f$ should satisfy (1.1). Using the definition of symmetric connections, we can also give a corresponding concept for interpolation functions and $n$-monotone functions. It is shown that the space of $n$-connections is strictly subset of the space of positive $n$-monotone functions on $(0, \infty)$ (Corollary 2.9). However, restricting on the symmetric functions, the space of symmetric $n$-monotone functions is the same as that of symmetric $n$ connections (Theorem 2.10). 


\section{Interpolation FUnCtions AND MeAns of POSitive MATrices}

In [12], there is an affine order-isomorphism from the set of connections onto the set of operator monotone functions. In this section, we describe the similar relation between the connections of order $n$ and $\mathcal{C}_{n} \supsetneq \mathcal{C}_{2 n}$. Note that every positive semi-definite matrix can be obtained as a limit of a decreasing sequence of positive definite matrices, from now on, we can always assume that connections are defined on positive definite matrices.

2.1. From $n$-connections to $P_{n}^{\prime}$. For any $n$-connection $\sigma$, the matrix $I_{n} \sigma\left(t I_{n}\right)$ is a scalar by $[12$, Theorem 3.2], and so we can define a function $f$ on $(0, \infty)$ by

$$
f(t) I_{n}=I_{n} \sigma\left(t I_{n}\right)
$$

where $I_{n}$ is the identity in $M_{n}$.

Claim: $f \in P_{n}^{\prime} \subsetneq \mathcal{C}_{n}$. Indeed, as in the proof of [12, Theorem 3.2], using the property (I) of the definition of connection, $f$ is a $n$-monotone function on $(0, \infty)$.

Injectivity: Let $\sigma_{1}$ and $\sigma_{2}$ be two $n$-connections. Then there correspond two functions $f_{1}$ and $f_{2}$ belonging to $\mathcal{C}_{n}$, where $f_{i}(t) I_{n}=I_{n} \sigma_{i}\left(t I_{n}\right)(i=1,2)$. Suppose that $f_{1}=f_{2}$ then we have, for any $A>0$ and $B>0$ of order $n$,

$$
\begin{aligned}
A \sigma_{1} B & =A^{\frac{1}{2}}\left(I_{n} \sigma_{1} A^{\frac{-1}{2}} B A^{\frac{-1}{2}}\right) A^{\frac{1}{2}} \quad([12,(3.8)]) \\
& =A^{\frac{1}{2}} f_{1}\left(A^{\frac{-1}{2}} B A^{\frac{-1}{2}}\right) A^{\frac{1}{2}} \\
& =A^{\frac{1}{2}} f_{2}\left(A^{\frac{-1}{2}} B A^{\frac{-1}{2}}\right) A^{\frac{1}{2}} \\
& =A \sigma_{2} B .
\end{aligned}
$$

Hence, $\sigma_{1}=\sigma_{2}$ by the continuity of means.

2.2. From $\mathcal{C}_{2 n}$ to $n$-connections. Let $f$ be a function belonging to $\mathcal{C}_{n}$. We can define a binary operation $\sigma$ on positive definite matrices in $M_{n}$ by:

$$
A \sigma B=A^{\frac{1}{2}} f\left[A^{\frac{-1}{2}} B A^{\frac{-1}{2}}\right] A^{\frac{1}{2}}, \quad \forall A, B>0 .
$$

This operation satisfies the property (III) of the definition of connection. Indeed, let $A_{n}$ and $B_{n}$ be two decreasing sequences which converge strongly to $A$ and $B$, respectively. Then $A_{n}^{-1}$ and $B_{n}^{-1}$ converge strongly to $A^{-1}$ and $B^{-1}$, respectively. Therefore, $A_{n}^{\frac{-1}{2}} B_{n} A_{n}^{\frac{-1}{2}}$ converges strongly to $A^{\frac{-1}{2}} B A^{\frac{-1}{2}}$ and by the continuity of $f$ we get the property (III). In [12], if $f$ is an operator monotone, then the operation $\sigma$ defined above can be represented as:

$$
A \sigma B=\int_{[0, \infty]} \frac{1+s}{s}\{(s A): B\} d \rho(s),
$$

where $\rho$ is the Radon measure on $[0, \infty]$ corresponding to $f$ (see $[12$, Theorem $3.4]$ ). Unfortunately, in the case $f$ belongs to $\mathcal{C}_{n}$ considered here, we do not know the existence of the measure $\rho$ satisfying the representation (2.2). However, we can have such the representation of $\sigma$ at "locally" as follows.

Lemma 2.1. Let $f$ be a function in $\mathcal{C}_{n}$ and $A, B$ positive matrices of order $n$. Then there exists a Radon measure on the spectrum of $A^{\frac{-1}{2}} B A^{\frac{-1}{2}}$ such that the binary operation $\sigma$ determined by (2.1) can be represented as the integral (2.2). 
Proof. By Theorem 1.4, there exists a Radon measure $\rho=\rho_{\sigma\left(A^{\frac{-1}{2}} B A^{\frac{-1}{2}}\right)}$ on $[0, \infty]$ such that

$$
f\left[A^{\frac{-1}{2}} B A^{\frac{-1}{2}}\right]=\int_{0}^{\infty} A^{\frac{-1}{2}} B A^{\frac{-1}{2}}(1+s)\left(A^{\frac{-1}{2}} B A^{\frac{-1}{2}}+s\right)^{-1} d \rho(s),
$$

where $\sigma\left(A^{\frac{-1}{2}} B A^{\frac{-1}{2}}\right)$ is the set of eigenvalues of $A^{\frac{-1}{2}} B A^{\frac{-1}{2}}$. Substituting this equality into (2.1), we have

$$
\begin{aligned}
A \sigma B & =A^{\frac{1}{2}} \int_{0}^{\infty}\left[A^{\frac{-1}{2}} B A^{\frac{-1}{2}}\right](1+s)\left(A^{\frac{-1}{2}} B A^{\frac{-1}{2}}+s\right)^{-1} d \rho(s) A^{\frac{1}{2}} \\
& =\int_{0}^{\infty} B A^{\frac{-1}{2}}(1+s)\left(A^{\frac{-1}{2}} B A^{\frac{-1}{2}}+s\right)^{-1} A^{\frac{1}{2}} d \rho(s) \\
& =\int_{0}^{\infty}(1+s)\left(A^{\frac{-1}{2}}\left(A^{\frac{-1}{2}} B A^{\frac{-1}{2}}+s\right) A^{\frac{1}{2}} B^{-1}\right)^{-1} d \rho(s) \\
& =\int_{0}^{\infty}(1+s)\left(A^{-1}+s B^{-1}\right)^{-1} d \rho(s) \\
& =\int_{0}^{\infty} \frac{1+s}{s}\{(s A): B\} d \rho(s) .
\end{aligned}
$$

Corollary 2.2. Let $f$ be a positive function on $(0, \infty)$ belonging to $\mathcal{C}_{n}$. Then there is a semi-connection of order $n, \sigma$, such that $f(t) I_{n}=I_{n} \sigma\left(t I_{n}\right)$ for $t>0$.

Proof. We can define a binary $\sigma$ by the formula (2.1). Because of the continuity of $f$ (see Remark 2.3 below), we imply that $\sigma$ has the property (III) in the definition. By Lemma 2.1, there exists a Radon measure $\rho$ such that

$$
A \sigma B=\int_{[0, \infty]} \frac{1+s}{s}\{(s A): B\} d \rho(s)
$$

For any positive definite matrix $C$ of order $n$,

$$
\begin{aligned}
C(A \sigma B) C & =\int_{[0, \infty]} \frac{1+s}{s} C\{(s A): B\} C d \rho(s) \\
& =\int_{[0, \infty]} \frac{1+s}{s}\{(s C A C): C B C\} d \rho(s) \\
& =(C A C) \sigma(C B C) .
\end{aligned}
$$

In the proof above, we need the continuity of $f \in \mathcal{C}_{n}$. Actually, we follow the definition of interpolation function in [4] and the continuity is the prior assumption for any function. However, even if we did not assume the continuity of the functions under consideration, we have

Remark 2.3. If $f \in \mathcal{C}_{n}(I)$ for $n>2$ then $f$ is continuous on $I$.

Proof. In order to prove the remark, we use the following facts.

(i) Any convex function on an open interval is continuous. (c.f. [15, Theorem 1.3.3]) We may assume that $I=(-1,1)$. 
(ii) If $f \in \mathcal{C}_{3}$, then $g(t)=(t+1) f(t)$ is convex (see the below), and $f$ is continous.

To prove the remark, we do the same step in the proof of [7, Theorem V. 3.6]. Indeed, since $f \in \mathcal{C}_{3}$, for a finite set $S$ of any three points $t_{1}, t_{2}, \lambda t_{1}+(1-\lambda) t_{2} \in I$ $(0<\lambda<1)$ there exists an operator monotone function $h$ such that $f=h$ on $S$. Since $g_{1}(t)=(t+1) h(t)$ is operator convex on $(-1,1)$ by [7, Lemma V. 3. 5], we have

$$
\begin{aligned}
g\left(\lambda t_{1}+(1-\lambda) t_{2}\right) & =g_{1}\left(\lambda t_{1}+(1-\lambda) t_{2}\right) \\
& \leq \lambda g_{1}\left(t_{1}\right)+(1-\lambda) g_{1}\left(t_{2}\right) \\
& =\lambda g\left(t_{1}\right)+(1-\lambda) g\left(t_{2}\right)
\end{aligned}
$$

and $g(t)=(t+1) f(t)$ is convex. So, $g(t)=(t+1) f(t)$ is continuous. Since $(t+1)$ is positive on $(-1,1), f$ is continuous on $(-1,1)$.

Now we can state the main theorem of this section.

Theorem 2.4. For any natural number $n$ there is an injective map $\Sigma$ from the set of matrix connections of order $n$ to $P_{n}^{\prime} \supset \mathcal{C}_{2 n}$ associating each connection $\sigma$ to the function $f_{\sigma}$ such that $f_{\sigma}(t) I_{n}=I_{n} \sigma\left(t I_{n}\right)$ for $t>0$. Furthermore, the range of this map contains $\mathcal{C}_{2 n}$.

Proof. We have only to prove that the range of the map $\Sigma$ contains $C_{2 n}$. For any $f \in \mathcal{C}_{2 n}$, since $\mathcal{C}_{2 n} \subset \mathcal{C}_{n}$, by Corollary 2.2, there is a semi-connection $\sigma_{f}$ defined by the formula $(2.1)$ and $f(t) I_{n}=I_{n} \sigma_{f}\left(t I_{n}\right)$ on $(0, \infty)$. Since $f \in \mathcal{C}_{2 n}$, by Theorem 1.4 we have that for any $0<A \leq C$ and $0<B \leq D$ there exists a Radon measure $\rho$ on $\sigma\left(A^{\frac{-1}{2}} B A^{\frac{-1}{2}}\right) \cup \sigma\left(C^{\frac{-1}{2}} D C^{\frac{-1}{2}}\right)$ such that

$$
\begin{aligned}
& A \sigma_{f} B=\int_{[0, \infty]} \frac{1+s}{s}\{(s A): B\} d \rho(s), \\
& C \sigma_{f} D=\int_{[0, \infty]} \frac{1+s}{s}\{(s C): D\} d \rho(s) .
\end{aligned}
$$

Since $\{(s A): B\} \leq\{(s C): D\}$, the condition (I) satisfies. Hence $\sigma_{f}$ is a connection of order $n$. Since $\Sigma\left(\sigma_{f}\right)(t) I_{n}=I_{n} \sigma_{f}\left(t I_{n}\right)=f(t) I_{n}$ for any $t \in \mathbb{R}^{+}$, we are done.

Remark 2.5. Since $P_{n}^{\prime} \subsetneq \mathcal{C}_{n}$, the map associating each connection of order $n$ to a function in $\mathcal{C}_{n}$ as above is not surjective.

2.3. Decreasing inclusion of the connections of order $n$. Via the usual embedding of $M_{n}$ into $M_{n+1}$, it is straightforward to check that the classes of connections of order $n$ is decreasing. It is natural to ask the following question: Is there a matrix mean $\sigma_{n}$ of the order $n$ on $M_{n}$ such that $\sigma_{n}$ is not of order $n+1$ ?

The following observation gives partially affirmative data to the above question.

Proposition 2.6. 
(1) For any $n \geq 2$ there is a matrix mean $\sigma_{n}$ of order $n$ which is not of order $n+2$.

(2) There is a matrix mean $\sigma_{1}$ of order 1 which is not of order 2.

Proof. (1): Take $f \in \mathcal{C}_{2 n} \backslash P_{n+2}^{\prime}$ (actually, we take $f \in P_{n+1}^{\prime} \backslash P_{n+2}^{\prime}$ ). Note that we can take such a function as $f(0)=0$. Then we have a matrix mean $\sigma_{f}$ of order $n$ such that $f(t) I_{n}=I_{n} \sigma_{f}\left(t I_{n}\right)$ for $t \in \mathbb{R}^{+}$by Theorem 2.4. Suppose on the contrary that $\sigma_{f}$ is a matrix mean of order $(n+2)$.

From Theorem 2.4 there is a $(n+2)$-monotone function $g$ such that $g(s) I_{n+2}=$ $I_{n+2} \sigma_{f}\left(s I_{n+2}\right)$ for $s \in \mathbb{R}^{+}$. For any $A \in M_{n}^{+}$we set $\tilde{A}=\operatorname{diag}\left(A, O_{2}\right) \in M_{n+2}^{+}$. Then $g(\tilde{A})=\operatorname{diag}\left(g(A), g\left(O_{2}\right)\right)$. Therefore

$$
\begin{aligned}
\operatorname{diag}\left(g(A), O_{2}\right) & =\operatorname{diag}\left(I_{n}, O_{2}\right) g(\tilde{A}) \operatorname{diag}\left(I_{n}, O_{2}\right) \\
& =\operatorname{diag}\left(I_{n}, O_{2}\right)\left(I_{n+2} \sigma_{f} \tilde{A}\right) \operatorname{diag}\left(I_{n}, O_{2}\right) \\
& =\operatorname{diag}\left(I_{n}, O_{2}\right) I_{n+2} \sigma_{f} \tilde{A} \operatorname{diag}\left(I_{n}, O_{2}\right) \quad([12,(3.6)]) \\
& =\operatorname{diag}\left(I_{n} f(A), O_{2}\right) \quad(f(0)=0) \\
& =\operatorname{diag}\left(f(A), O_{2}\right)
\end{aligned}
$$

This means that $f(x)=g(x)$ for $x \in \mathbb{R}^{+}$, hence $f \in P_{n+2}^{\prime}$. This is a contradiction to the assumption that $f \notin P_{n+2}^{\prime}$.

(2): Take $f \in \mathcal{C}_{2} \backslash P_{2}^{\prime}$ (see [4, Proposition 3.4]).

From Corollary 3.3 there is a mean $\sigma_{f}$ of order 1 . We know, then, $\sigma_{f}$ is not of order 2. Indeed, if $\sigma_{f}$ is of order 2, there is a 2-monotone $h$ such that $h(t) I_{2}=I_{2} \sigma_{f}\left(t I_{2}\right)$ from the argument in Section 3.1. Then since $f(t)=h(t)$ for $t \in R^{+}, f$ is 2-monotone, and a contradiction. Therefore, $\sigma_{f}$ is not of order 2 .

We can give here another proof of Proposition 2.6.

Proof. Denote by $\Sigma_{n}$ the image of the class of connections of order $n$ via the map in Theorem 2.4 for each $n$. Therefore, $\Sigma_{n}$ is isomorphic to the class of $n$ connections (so the sequence $\left\{\Sigma_{n}\right\}$ is decreasing) and $\Sigma_{n} \subseteq P_{n}^{\prime}$. From now on, we will identify the space of $n$-connections with $\Sigma_{n}$.

(1): On account of Remark 1.2 and Theorem 2.4, we obtain the following inclusion:

$$
\begin{aligned}
\Sigma_{n+2} & \subseteq P_{n+2}^{\prime} \subseteq \mathcal{C}_{2(n+1)+1} \subseteq \mathcal{C}_{2(n+1)} \subseteq \Sigma_{n+1} \\
& \subseteq P_{n+1}^{\prime} \subseteq \mathcal{C}_{2 n+1} \subseteq \mathcal{C}_{2 n} \subseteq \Sigma_{n} .
\end{aligned}
$$

And since $P_{n+2}^{\prime} \subsetneq P_{n+1}^{\prime}$, we imply that $\Sigma_{n+2} \subsetneq \Sigma_{n}$.

(2): Using Remark 1.2 again and Corollary 3.3, we get

$$
\Sigma_{2} \subseteq P_{2}^{\prime} \subseteq \mathcal{C}_{3} \subseteq \mathcal{C}_{2}=\Sigma_{1}
$$

By $P_{2}^{\prime} \neq \mathcal{C}_{3}[4$, Proposition 3.14], we then have the statement.

Remark 2.7. From the second proof of Proposition 2.6, we highlight the inclusion: For each natural number $n$,

$$
\mathcal{C}_{2(n+1)} \subseteq \Sigma_{n+1} \subseteq P_{n+1}^{\prime} \subseteq \mathcal{C}_{2 n+1} \subseteq \mathcal{C}_{2 n} \subseteq \Sigma_{n} \subseteq P_{n}^{\prime}
$$


2.4. Symmetric connections. As the same in [12], we can recall some notations and properties of connections as follows. Let $\sigma$ be a $n$-connection. The transpose $\sigma^{\prime}$, the adjoint $\sigma^{*}$ and the dual $\sigma^{\perp}$ of $\sigma$ are defined by

$$
A \sigma^{\prime} B=B \sigma A, \quad A \sigma^{*} B=\left(A^{-1} \sigma B^{-1}\right)^{-1}, \quad \sigma^{\perp}=\sigma^{*} .
$$

A connection is called symmetric if it equals to its transpose. Denoted by $\Sigma_{n}^{s y m}$ the set of $n$-monotone representing functions of symmetric $n$-connections, i.e., $\Sigma_{n}^{s y m}$ is the image of the set of all symmetric $n$-connections via the canonical map in Theorem 2.4. Then, using the same argument as in [12], we can state the following properties for any $n$-connection:

(1) $\sigma+\sigma^{\prime}$ and $\sigma(:) \sigma^{\prime}$ are symmetric.

(2) $\omega_{l}(\sigma) \omega_{r}=\sigma ; \omega_{r}(\sigma) \omega_{l}=\sigma^{\prime}$, where $A \omega_{l} B=A$ and $A \omega_{r} B=B$.

(3) The $n$-monotone representing function of the $n$-connection $\sigma(\tau) \rho$ is indeed $f(x) g[h(x) / f(x)]$, where $f, g, h$ are the representing functions of $\sigma, \tau, \rho$ in Theorem 2.4, respectively.

(4) $\sigma$ is symmetric if and only if its $n$-monotone representing function $f$ is symmetric, that is, $f(x)=x f\left(x^{-1}\right)$.

Each $n$-connection corresponds to a positive $n$-monotone function belonging to $\Sigma_{n}$ by Theorem 2.4. Therefore, combining with the observation above, we get the following.

Proposition 2.8. Let $f(x), g(x), h(x)$ belong to $\Sigma_{n}$. Then the following statements hold true:

(i) $k(x)=x f\left(x^{-1}\right), f^{*}(x)=f\left(x^{-1}\right)^{-1}, \frac{x}{f(x)}, f(x) g[h(x) / f(x)], a f(x)+b g(x)$ all belong to $\Sigma_{n}$;

(ii) $f(x)+k(x), \frac{f(x) k(x)}{f(x)+k(x)}$ all belong to $\Sigma_{n}^{s y m}$.

Proof. By the hypothesis, there are $n$-connections $\sigma, \tau, \rho$ such that their representing functions are $f(x), g(x), h(x)$, respectively. Then the statements follow from the the fact that the functions $k(x)=x f\left(x^{-1}\right), f^{*}(x)=f\left(x^{-1}\right)^{-1}, \frac{x}{f(x)}$,

$a f(x)+b g(x), f(x) g[h(x) / f(x)], f(x)+k(x), \frac{f(x) k(x)}{f(x)+k(x)}$ are the representing functions of $n$-connections $\sigma^{\prime}, \sigma^{*}, \sigma^{\perp}, a \sigma+b \tau, \sigma(\tau) \rho, \sigma+\sigma^{\prime}, \sigma(:) \sigma^{\prime}$, respectively.

Corollary 2.9.

$$
\mathcal{C}_{2 n} \subseteq \Sigma_{n} \subsetneq P_{n}^{\prime}
$$

Proof. We only need to show that $\Sigma_{n} \neq P_{n}^{\prime}$ for $n>1$. Suppose on the contrary that $\Sigma_{n}=P_{n}^{\prime}$. Let

$$
p(x)=\sum_{k=1}^{2 n-1} \frac{1}{k !} x^{k} .
$$

Then $p(x)$ belongs to $P_{n}^{\prime}\left(0, \alpha_{n}\right)$ for some $\alpha_{n}>0$ (see [13]). Let $\phi$ be the operator monotone isomorphism from $\left(0, \alpha_{n}\right)$ to $(0, \infty)$ defined by

$$
\phi(x)=\frac{x}{\alpha_{n}-x} .
$$

Then $p \circ \phi^{-1}$ belongs to $P_{n}^{\prime}$. By the assumption, $p \circ \phi^{-1} \in \Sigma_{n}$. Then 


$$
x\left(p \circ \phi^{-1}\right)\left(x^{-1}\right)=x p\left(\frac{\alpha_{n}}{1+x}\right)=\sum_{k=1}^{2 n-1} \frac{\alpha_{n}^{k}}{k !} \frac{x}{(1+x)^{k}}
$$

is in $\Sigma_{n}$ by Proposition 2.8. In particular, $x p\left(\frac{\alpha_{n}}{1+x}\right)$ is monotone; this is impossible if $n>1$. Indeed, the first derivative of the function $\frac{x}{(1+x)^{k}}$ is $\frac{1+(1-k) x}{(1+x)^{k+1}}$ and is negative for sufficiently large $x$ when $k \geq 2$.

But if we restrict our attention to the class of the symmetric, we get the following equality.

\section{Theorem 2.10.}

$$
\Sigma_{n}^{s y m}=P_{n}^{\text {sym }}
$$

where $P_{n}^{\prime s y m}$ is the set of all symmetric functions in $P_{n}^{\prime}$.

Proof. The inclusion $\Sigma_{n}^{s y m} \subset P_{n}^{\text {sym }}$ is trivial by Theorem 2.4.

Let $f$ be a symmetric function in $P_{n}^{\prime}$. We can define a binary operation on positive definite matrices of order $n$ by

$$
A \sigma B=A^{\frac{1}{2}} f\left[A^{\frac{-1}{2}} B A^{\frac{-1}{2}}\right] A^{\frac{1}{2}} .
$$

For any $B \leq D$, then $A^{\frac{-1}{2}} B A^{\frac{-1}{2}} \leq A^{\frac{-1}{2}} D A^{\frac{-1}{2}}$. Since $f$ is $n$-monotone and the conjugate action preserves the order on self-adjoint matrices, we obtain

$$
A^{\frac{1}{2}} f\left[A^{\frac{-1}{2}} B A^{\frac{-1}{2}}\right] A^{\frac{1}{2}} \leq A^{\frac{1}{2}} f\left[A^{\frac{-1}{2}} D A^{\frac{-1}{2}}\right] A^{\frac{1}{2}} .
$$

This means $A \sigma B \leq A \sigma D$. Since $f$ is symmetric, we also have

$$
A \sigma D=D^{\frac{1}{2}} f\left[D^{\frac{-1}{2}} A D^{\frac{-1}{2}}\right] D^{\frac{1}{2}} .
$$

Using this identity, we can also show that $A \sigma D \leq C \sigma D$ whenever $A \leq C$. Thus, $A \sigma B \leq A \sigma D \leq C \sigma D$ for any positive matrices $A, B, C, D$ with $A \leq C$ and $B \leq D$.

Remark 2.11. We would like to mention that even $P_{n+1}^{\prime} \subsetneq P_{n}^{\prime}$, but we still do not know whether $P_{n+1}^{\text {sym }} \subsetneq P_{n}^{\prime s y m}$ holds or not. As the first thought, we can obtain a symmetric function from the polynomial in $P_{n+1}^{\prime}$ but not in $P_{n}^{\prime}$ and such a function is a candidate to show $P_{n+1}^{\prime s y m} \subsetneq P_{n}^{\prime s y m}$. Unfortunately, this is not true as the following example.

Example 2.12. Let $p(x)=x+\frac{1}{2} x^{2}+\frac{1}{6} x^{3}$ be a polynomial which belongs to $P_{2}^{\prime}(0, \alpha)$ but does not belong to $P_{3}^{\prime}(0, \alpha)$ for some $\alpha>0$ (see [13]). Let $q(x)$ be the symmetrization of $p$ by

$$
q(x)=p(x)+x p\left(x^{-1}\right) .
$$

Then $q$ is symmetric. However, we can show that $q$ does not belong to $P_{2}^{\prime}(0, \alpha)$. Indeed, the matrix

$$
\left(\begin{array}{cc}
q^{\prime}(x) & \frac{1}{2} q^{\prime \prime}(x) \\
\frac{1}{2} q^{\prime \prime}(x) & \frac{1}{6} q^{\prime \prime \prime}(x)
\end{array}\right)
$$

is not positive semi-definite for every $x>0$. 
Remark 2.13. Note that a function $f$ on an interval $I$ is $n$-monotone if and only if the $n \times n$ matrix

$$
\left[f^{(i+j-1)}(t) /(i+j-1) !\right]
$$

is positive for any $t \in I$ (for example see [10, VII Theorem VI and VIII Theorem $\mathrm{V}]$ ).

\section{TOWARD THE CONJECTURE $\mathcal{C}_{2 n}=\Sigma_{n}$}

We know that $\mathcal{C}_{2 n} \subseteq \Sigma_{n} \subseteq P_{n}^{\prime}$ and $\mathcal{C}_{2}=\Sigma_{1}$ (see Corollary 3.3). Therefore, we may give a conjecture that, for any positive integer $n$,

$$
\mathcal{C}_{2 n}=\Sigma_{n} \text { and } \Sigma_{n}^{s y m}=\mathcal{C}_{2 n}^{s y m}
$$

Even we still do not know whether $\mathcal{C}_{2 n}=\Sigma_{n}$ or not, but they have some similar properties. In particular, the properties of the space $\Sigma_{n}$ represented in Proposition 2.8 also hold true when we replace $\Sigma_{n}\left(\right.$ resp. $\Sigma_{n}^{s y m}$ ) by $\mathcal{C}_{2 n}$ (resp. $\left.C_{2 n}^{s y m}\right)$. That is,

Proposition 3.1. The statements in Proposition 2.8 hold if we replace $\Sigma_{n}$ (resp. $\Sigma_{n}^{\text {sym }}$ ) by $\mathcal{C}_{2 n}\left(\right.$ resp. $\left.C_{2 n}^{\text {sym }}\right)$.

Proof. (i): Let $S$ be a subset of $(0, \infty)$ consisting $2 n$ points. There exists an operator monotone function $p(x)$ such that $p$ are identified with $f$ on $S$. Set $p_{1}(x)=p\left(x^{-1}\right)^{-1}$, then $p_{1}$ is an operator monotone function and $p_{1}$ equals to $f^{*}$ on $S$. Hence, the function $x / k(x)=f^{*}(x) \in \mathcal{C}_{2 n}$. This implies that $k(x)$ belongs to $\mathcal{C}_{2 n}$ by Remark $1.2(\mathrm{v})$. It is routine to check that af $(x)+b g(x)$ belongs to $\mathcal{C}_{2 n}$.

In order to show that $f(x) g[h(x) / f(x)]$ belongs to $\mathcal{C}_{2 n}$, by Theorem 1.3 , we have only to show that this function is equal to an operator monotone function on any $2 n$-point subset $S$ of $(0, \infty)$. Since $f, g, h$ belong to $\mathcal{C}_{2 n}$, they are identified with operator monotone functions on $S$, without confusing let us still assume that these monotone functions are $f, g, h$ respectively. Therefore, in order to complete the proof, we will show that the function $f(x) g[h(x) / f(x)]$ is operator monotone whenever $f, g, h$ are operator monotone. Indeed, the function $f(x) g[h(x) / f(x)]$ was taken up as an issue of practice to be operator monotone due to [12, Theorem 3.2 and Lemma 4.1]. However, we can give here a more elementary proof by using the fact that a positive function $F$, which is strictly positive on $\mathbb{R}^{+}$is operator monotone if and only if $0<\arg F(z) \leq \arg z$ for any $z$ in the upper half plane. This comes from [7, V(53)] and from the fact that $0<\arg (z+a)<\arg (z)$ for $a>0$ and $z$ in the upper half plane. Note that $-\pi<\arg \frac{h(z)}{f(z)}<\pi$ if $0<\arg z<\pi$. 
When $0<\arg \frac{h(z)}{f(z)}<\pi$, we have

$$
\begin{aligned}
0<\arg f(z) g\left(\frac{h(z)}{f(z)}\right) & =\arg f(z)+\arg g\left(\frac{h(z)}{f(z)}\right) \\
& \leq \arg f(z)+\arg \frac{h(z)}{f(z)} \\
& \leq \arg f(z)+\arg h(z)-\arg f(z) \\
& \leq \arg h(z) \\
& \leq \arg (z) .
\end{aligned}
$$

When $-\pi<\arg \frac{h(z)}{f(z)}<0$, we have

$$
\begin{aligned}
0<\arg h(z) & =\arg f(z)+\arg \frac{h(z)}{f(z)} \\
& \leq \arg f(z)+\arg g\left(\frac{h(z)}{f(z)}\right) \\
& =\arg f(z) g\left(\frac{h(z)}{f(z)}\right) \\
& <\arg f(z)<\pi .
\end{aligned}
$$

Hence $f(x) g\left(\frac{h(x)}{f(x)}\right)$ belongs to $\mathcal{C}_{2 n}$.

(ii): If $f(x) \in \mathcal{C}_{2 n}$, by (i), $k(x) \in \mathcal{C}_{2 n}$ and hence $f(x)+k(x)$ belongs to $\mathcal{C}_{2 n}^{\text {sym }}$. To show that $\frac{f(x) k(x)}{f(x)+k(x)}$ belongs to $\mathcal{C}_{2 n}^{\text {sym }}$, we apply the fact from (i) that $f(x) g[h(x) / f(x)]$ belongs to $\mathcal{C}_{2 n}$ with $g(x)=x /(1+x)$ and $h(x)=k(x)$.

Note that Proposition 3.1 still holds true in the space $\mathcal{C}_{n}$.

We have the application of Proposition 3.1 to the following well-known result (see [7, Exercise V. 4.15]).

Corollary 3.2. If a polynomial of degree $m$

$$
p(x)=\sum_{i=0}^{m} a_{i} x^{i}, \quad a_{m} \neq 0
$$

belongs to $P^{\prime}$, then $m \leq 1$.

Proof. Since $p$ is monotone, $a_{m}>0$. A function in $P^{\prime}$ belongs to $\mathcal{C}_{2 n}$ for every $n$, so by Proposition 3.1, $x p\left(x^{-1}\right)$ also belongs to $\mathcal{C}_{2 n}$ for every $n$. Hence, $x p\left(x^{-1}\right)$ belongs to $P^{\prime}$. This implies that $x p\left(x^{-1}\right)$ is monotone and this property holds only when the degree of $p(x)$ is not more than 1 .

3.1. Matrix means of order one. We recall the results in [4] for the sets $\mathcal{C}_{1}, \mathcal{C}_{2}$ as follows.

- $\mathcal{C}_{1}$ is the set of all positive functions on $(0, \infty)$.

- $\mathcal{C}_{2}$ consists of all quasi-concave functions (i.e., $f(s) \leq f(t) \max \left\{1, \frac{s}{t}\right\}$ for all $s, t>0)$. 
For any connection $\sigma$ of order 1 , then the corresponding function $f$ belongs to $\mathcal{C}_{2}$. Indeed, for any numbers $0<t \leq s$, we have

$$
\begin{aligned}
f(t) \max \left\{1, \frac{s}{t}\right\} & =(1 \sigma t) \frac{s}{t}=\frac{s}{t} \sigma s \\
& \geq 1 \sigma s=f(s), \text { and } \\
f(s) \max \left\{1, \frac{t}{s}\right\} & =(1 \sigma s) \\
& \geq 1 \sigma t=f(t) .
\end{aligned}
$$

Combining this property with Theorem 2.4, we obtain:

\section{Corollary 3.3.}

(1) Every connection $\sigma$ of order 1 can be determined uniquely by

$$
x \sigma y=x f\left(\frac{y}{x}\right) \quad \forall x, y>0,
$$

where $f$ is an interpolation function in $\mathcal{C}_{2}$.

(2) Every function $f$ in $\mathcal{C}_{2}$ can be represented uniquely by

$$
f(x)=1 \sigma x \quad \forall x>0,
$$

where $\sigma$ is a connection of order 1 .

From this corollary, we can easily get the functions in $\mathcal{C}_{2}$ from the corresponding connections and vise versa. For example, the functions in $\mathcal{C}_{2}$ which correspond to

arithmetic mean, harmonic mean and the geometric mean are $\frac{1+x}{2}, \frac{2}{1+x}$ and $x^{\frac{1}{2}}$; and any (positive) linear combination of these functions also belongs to $\mathcal{C}_{2}$.

If we take the function $f(x)=2 \frac{x}{1+x}+\left(\frac{x}{1+x}\right)^{2} \in \mathcal{C}_{2} \backslash \mathcal{C}_{3}$ in [4, Example 3.13], we have a connection $\sigma_{f}$ of order 1 which is not of order 2 as follows:

$$
\begin{aligned}
x \sigma_{f} y & =x f\left(\frac{y}{x}\right) \\
& =2 \frac{x y}{x+y}+\frac{x y^{2}}{(x+y)^{2}}
\end{aligned}
$$

for $x, y \in \mathbb{R}^{+}$.

Acknowledgement. The third author would like to appreciate Masaru Nagisa who kindly gives an admission to cite his beautiful proof of Proposition 3.1(ii). Research partially supported by JSPS KAKENHI Grant Number 20540220, 26400125 and by NAFOSTED, Vietnam, Grant No. 101.04.2014.40.

\section{REFERENCES}

1. E.Y. Ameur, Interpolation of Hilbert spaces, Thesis (Ph.D.)Uppsala Universitet (Sweden). ProQuest LLC, Ann Arbor, MI, 2002.

2. Y. Ameur, The Calderon problem for Hilbert couples, Ark. Math. 41 (2003), no. 2, 203-231.

3. E.Y. Ameur, A new proof of Donoghue's interpolation theorem, J. Funct. Spaces Appl. 2 (2004), no. 3, 253-265.

4. Y. Ameur, S. Kaijser and S. Silvestrov, Interpolation class and matrix monotone functions, J. Operator Theory. 52 (2007), 409-427. 
5. W.N. Anderson, Jr. and R.J. Duffin, Series and parallel addition of matrices, J. Math. Anal. Appl. 26 (1969), 576-594.

6. W.N. Anderson, Jr. and G.E. Trapp, Shorted Operators II, Siam J. Appl. Math. 28 (1975), $60-71$.

7. R. Bhatia, Matrix Analysis, Springer-Verlag, New York, 1986.

8. W.F. Donoghue, The theorems of Loewner and Pick, Israel J. Math. 4 (1966), 153-170.

9. W.F. Donoghue, The interpolation of quadratic norms, Acta Math. 118 (1967), 251-270.

10. W.F. Donoghue, Monotone matrix function and analytic continuation, Springer 1974.

11. C. Foias and J. L. Lions, Sur certains theoremes d'interpolation, Acta Sci. Math (Szeged). 22 (1961), 269-282.

12. F. Kubo and T. Ando, Means of positive linear operators, Math. Ann. 246 (1980), 205-224.

13. F. Hansen, G. Ji and J. Tomiyama, Gaps between classes of matrix monotone functions, Bull. London Math. Soc. 36 (2004), 53-58.

14. K. Löwner, Über monotone matrixfunktionen, Math. Z. 38 (1934), 177-216.

15. C. Niculescu and L.-E.Persson, Convex functions and their applications, CMS Books in Mathematics/Ouvrages de Mathematiques de la SMC, 23. Springer, New York, 2006.

16. H. Osaka and J. Tomiyama, Note on the structure of matrix monotone functions, Analysis for Sciences, Engineering and Beyond, The tribute workshop in honor of Gunnar Sparr held in Lund, May 8-9, Spring Proceedings in Mathematics, 6 (2008), 319-324.

17. D. Petz, Monotone metric on matrix spaces, Linear Algebra Appl. 244 (1996), 81-96.

${ }^{1}$ Computational Mathematics and Engineering (CME), Institute for Computational Science (incos), Ton Duc Thang University, Ho Chi Minh City, Vietnam and Faculty of Civil Engineering, Ton Duc Thang University, Ho Chi Minh City, Vietnam for Computational Science (INCOS) \& Faculty of Civil Engineering, Ton Duc Thang University, Vietnam;

Department of Fundamental Sciences, Ho Chi Minh City University of Food InDustry, Ho Chi Minh City, Vietnam.

E-mail address: dinhtrunghoa@tdt.edu.vn; trunghoa.math@gmail.com

${ }^{2}$ Duy Tan University, Danang and Institute of Mathematics, VAST, 18 Hoang Quoc Viet, Hanoi, Vietnam.

E-mail address: hmtoan@math.ac.vn

3 Department of Mathematical Sciences, Ritsumeikan University, Kusatsu, Shiga 525-8577, JAPAN.

E-mail address: osaka@se.ritsumei.ac.jp 